\title{
The Vector Population Monitoring Tool (VPMT): High-Throughput DNA-Based Diagnostics for the Monitoring of Mosquito Vector Populations
}

\author{
Chris Bass, ${ }^{1}$ Dimitra Nikou, ${ }^{2}$ John Vontas, ${ }^{3}$ Martin J. Donnelly, ${ }^{2}$ Martin S. Williamson, ${ }^{1}$ \\ and Linda M. Field ${ }^{1}$ \\ ${ }^{1}$ Department of Biological Chemistry, Rothamsted Research, Harpenden AL5 2JQ, UK \\ ${ }^{2}$ Vector Group, Liverpool School of Tropical Medicine, Pembroke Place, Liverpool L3 5QA, UK \\ ${ }^{3}$ Department of Biology, University of Crete, Heraklion 71409, Greece \\ Correspondence should be addressed to Chris Bass, chris.bass@bbsrc.ac.uk
}

Received 28 September 2010; Accepted 17 November 2010

Academic Editor: Joao Pinto

Copyright (C 2010 Chris Bass et al. This is an open access article distributed under the Creative Commons Attribution License, which permits unrestricted use, distribution, and reproduction in any medium, provided the original work is properly cited.

Regular monitoring of mosquito vector populations is an integral component of most vector control programmes. Contemporary data on mosquito species composition, infection status, and resistance to insecticides are a prerequisite for effective intervention. For this purpose we, with funding from the Innovative Vector Control Consortium (IVCC), have developed a suite of highthroughput assays based on a single "closed-tube" platform that collectively comprise the "Vector Population Monitoring Tool" (VPMT). The VPMT can be used to screen mosquito disease vector populations for a number of traits including Anopheles gambiae s.l. and Anopheles funestus species identification, detection of infection with Plasmodium parasites, and identification of insecticide resistance mechanisms. In this paper we focus on the Anopheles-specific assays that comprise the VPMT and include details of a new assay for resistance to dieldrin $R d l$ detection. The application of these tools, general and specific guidelines on their use based on field testing in Africa, and plans for further development are discussed.

\section{Introduction}

A key component of most malaria control strategies is effective control of the mosquito vector. Indeed the World Health Organization supports vector control as the most generally effective measure to prevent malaria transmission and promotes a strategic approach to vector control known as Integrated Vector Management (IVM) [1]. IVM is defined as "a rational decision-making process for the optimal use of resources in the management of vector populations, so as to reduce or interrupt transmission of vector-borne diseases" [1]. An essential part of IVM is the gathering of contemporary information on vector populations. Data, such as which mosquito species occur in the local population, their infection status, and whether they are resistant to insecticides are essential for effective intervention strategies. This type of monitoring requires high-throughput sensitive diagnostic assays, and a large number of different methods have been described for this purpose. In many cases, these methods have garnered wide-spread acceptance and are now considered "gold standards"; however, in other cases, potentially serious deficiencies have been described $[2,3]$. In addition, previous methods have been based on a multitude of platforms including, but not limited to, allele-specific PCR (ASPCR), PCR-RFLP, Enzyme-linked Immunosorbent Assay (ELISA), Sequence Specific Oligonucleotide Probe-EnzymeLinked Immunosorbent Assay (SSOP-ELISA), PCR-Dot Blot and Heated Oligonucleotide Ligation Assay (HOLA), and this complicates their integrated use [4].

To address this, the Technology Development Group of the Innovative Vector Control Consortium has developed simple, high-throughput diagnostic assays to gather data on the status of mosquito vector populations [5]. The Anopheles-specific assays that comprise the "Vector Population Monitoring Tool" (VPMT) consist of a suite of highthroughput assays based on a single platform that can be used 
to screen vector populations for a number of traits. These include (1) Anopheles gambiae s.l. and Anopheles funestus species identification, (2) detection of infection with Plasmodium parasites, and (3) detection of insecticide resistance mechanisms [4, 6-10]. The latter currently consists of three mechanism-specific assays and includes (1) detection of knock-down resistance (kdr) mutations, (2) detection of insensitive acetylcholinesterase (iAChE), and (3) detection of resistance to dieldrin ( $\mathrm{Rdl})$. The assays that constitute the VPMT have been designed to be high throughput and require very small amounts of starting material (DNA) that can be extracted from mosquitoes that have been stored in a wide variety of ways (e.g., in ethanol, isopropanol, dried, and frozen). This ensures that they can be used for assaying field-collected mosquitoes without the need for a cold chain to preserve the specimens.

The assays are based on TaqMan SNP genotyping, a PCR method employing oligonucleotide probes that are dual labelled with a fluorescent reporter dye and a quencher molecule. As shown in Figure 1, amplification of the probespecific product causes cleavage of the probe, generating an increase in reporter fluorescence as the reporter dye is released away from the quencher. By using different reporter dyes, cleavage of allele-specific probes can be detected in a single PCR [11]. The "closed-tube" nature of the TaqMan platform means that there is no requirement for post-PCR processing and consequently assays are simple to perform and rapid to run.

In this paper we detail the assays that comprise the Anopheles-specific VPMT and provide advice on their use based on field testing of assays in Africa by ourselves and others. Finally, we discuss the application of these tools, the advantages and disadvantages of the platform on which they are based, and future development.

\section{Species Identification}

Identification of the species in the Anopheles gambiae sensu lato (s.l.) complex and the Anopheles funestus group is an important part of vector control programmes as both groups comprise species with different efficiencies as vectors and the distribution of different members often overlap.

\section{An. gambiae S.L. Species Complex}

The An. gambiae sensu lato (s.l.) species complex comprises seven species of mosquitoes that are morphologically indistinguishable but differ in their behaviour and ability to vector malaria. Anopheles gambiae sensu stricto s.s. and Anopheles arabiensis are the two principal vectors, whilst two other members Anopheles melas, and Anopheles merus are both saltwater breeding and consequently only important vectors in coastal regions $[12,13]$. Of the remaining members, Anopheles quadriannulatus species A and Anopheles quadriannulatus species B are considered to be zoophilic non-malaria vectors $[14,15]$, while Anopheles bwambae is restricted to a region close to the Buranga hot springs in Uganda [16].

Two alternative TaqMan assays for identification of members of this species complex have been developed as part

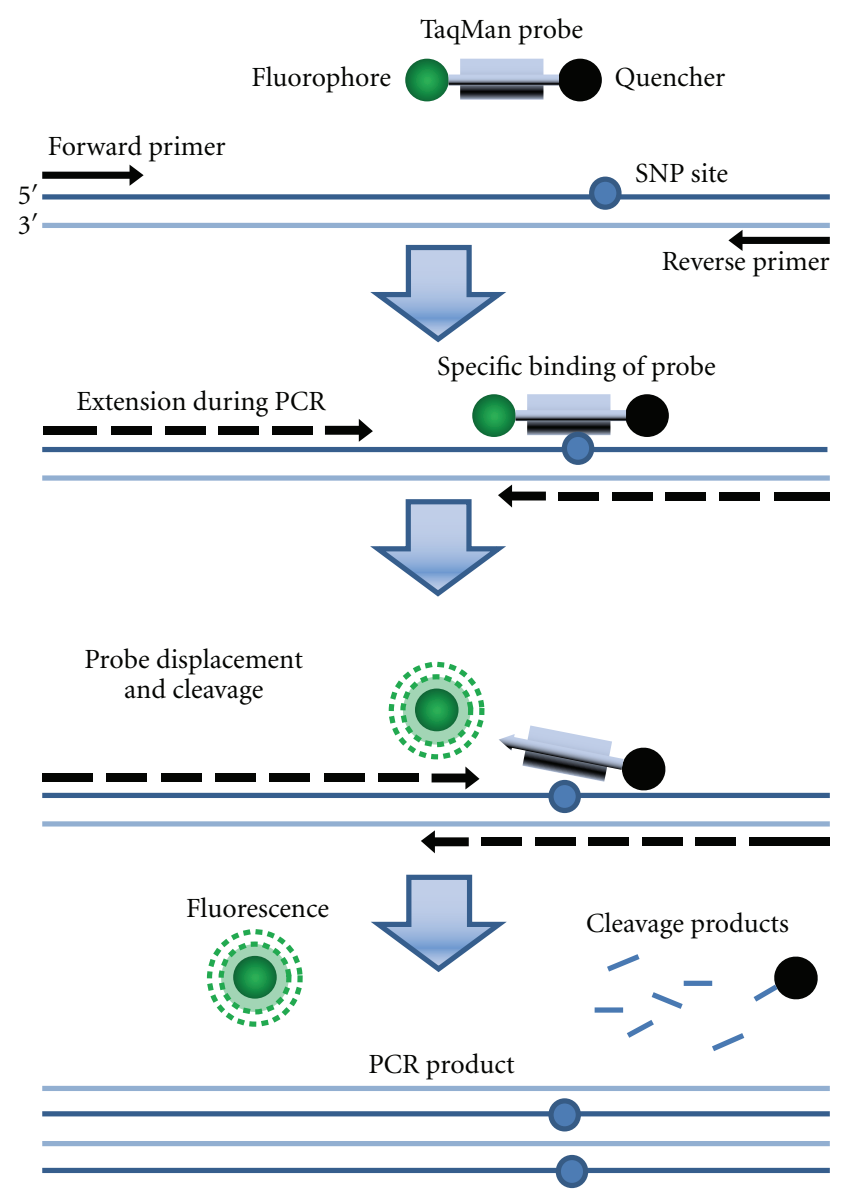

Figure 1: TaqMan SNP genotyping. The TaqMan assay is a PCR method employing oligonucleotide probes that are dual labelled with a fluorescent reporter dye and a quencher molecule. Amplification of the probe-specific product causes cleavage of the probe, generating an increase in reporter fluorescence as the reporter dye is released away from the quencher.

of the VPMT. The first [9] uses two probes to distinguish between the main malaria vectors An. gambiae s.s. and An. arabiensis as one group (detected by a probe labelled with the fluorophore FAM) and An. quadriannulatus, An. melas or An. merus as a second group (detected by a probe labelled with the fluorophore VIC). This assay can be used alone to discriminate vector from nonvector (in regions where $A n$. merus/melas/bwambae are not present) or in combination with an existing TaqMan assay [17] to further distinguish An. arabiensis from An. gambiae s.s.. The performance of this assay was compared with the "gold standard" AS-PCR method in a blind species identification trial of over 450 specimens collected from 13 countries in Sub-Saharan Africa [9]. The results showed that the TaqMan assay is comparable in specificity to standard PCR but is more sensitive, due to a lower detection threshold, and this results in a significantly lower rate of "failed reactions" (samples that cannot be identified due to low or no amplification). An advantage of this assay is its compatibility with real-time PCR machines that have two detection channels that are generally lower in 
cost than machines with three or more detection channels. A disadvantage of this assay is that, when used alone, it cannot discriminate between the two main vector species, An. gambiae s.s. and An. arabiensis. Therefore, a second assay [8] that overcomes this limitation was developed that uses three probes (labelled with Cy5, VIC, and FAM, resp.) to distinguish between An. arabiensis, An. gambiae s.s., and An. quadriannulatus/merus/melas/bwambae as a group. This assay requires a real-time PCR machine that has at least three detection channels. A genotyping trial with the sample set described above showed the new multiplex TaqMan assay to have a specificity and sensitivity comparable to the 2plex TaqMan assay [8]. A disadvantage of this assay is its limited use in regions where An. merus and An. melas are important local vector species and occur in sympatry with An. quadriannulatus. For this reason a future priority is to develop an assay that is able to discriminate these three species for application in such regions.

An. gambiae s.s. is currently considered to be undergoing speciation into two molecular forms (M and S). However recent studies of the genetic divergence of the two forms have indicated that this process may be more advanced than previously thought $[18,19]$. The current TaqMan assays do not discriminate between molecular forms of An. gambiae s.s. and a high-throughput assay, for this purpose is, therefore, an important future objective.

\section{An. funestus Species Complex}

The Anopheles funestus group consists of five subgroups (1) Anopheles funestus, (2) Anopheles rivulorum, (3) Anopheles minimus, (4) Anopheles aconitus, and (5) Anopheles culicifacies [20-22]. The African mosquito species An. funestus s.s., Anopheles parensis, and Anopheles vaneedeni, belonging to the An. funestus subgroup, and Anopheles leesoni and Anopheles rivulorum, belonging to the An. minimus and An. rivulorum subgroups, respectively, are either morphologically identical or very similar and may occur in sympatry over large parts of their distribution [22]. The vectorial capacity, biology, and behaviour of these species differ. Anopheles funestus s.s. is one of the most important African vectors of malaria [21] as a result of its anthropophilic and endophilic behaviour. The other species are predominantly zoophilic and exophilic $[20,21]$ and are thought to have limited, or no, importance as malaria vectors, although $A n$. vaneedeni has been shown to be susceptible to Plasmodium infection under laboratory conditions [23, 24] and An. rivulorum has been found infected with the Plasmodium parasite at one locality in Tanzania [25].

A multiplex TaqMan assay was, therefore, developed that detects and discriminates An. parensis, An. leesoni, An. vaneedeni, An. rivulorum, and An. funestus s.s [10]. The assay uses five probes labelled at the $5^{\prime}$ end with VIC for $A n$. parensis detection, 6FAM for An. leesoni detection, Cy5 for An. rivulorum detection, ROX for An. funestus s.s. detection, and Quasar 705 for An. vaneedeni detection. These five fluorophores have distinct emission and excitation spectra allowing their independent detection in a single reaction. In a blind trial, the TaqMan assay proved to be at least as sensitive and specific as the "gold standard" AS-PCR approach [10]. A disadvantage of the TaqMan assay is the requirement for a five-channel real-time PCR machine however, it may not be necessary for end users to test for all species. For example, it may not be necessary to test for An. vaneedeni in many SubSaharan countries as its distribution is limited to a localised region in Northern South Africa [20, 21].

\section{Plasmodium Infection}

An essential component of malaria vector control and monitoring programmes is the sensitive detection of the presence of the four human-specific Plasmodium species (Plasmodium falciparum, Plasmodium vivax, Plasmodium ovale and Plasmodium malariae) in the mosquito host. The infection status of a mosquito is usually assessed by the presence/absence of Plasmodium sporozoites in the salivary glands.

A TaqMan assay was developed that detects all four malaria-causing Plasmodium species and discriminates $P$. falciparum from $P$. vivax, $P$. ovale and $P$. malariae [6]. The assay uses two probes, the first labelled with FAM detects P. falciparum and the second, labelled with VIC detects $P$. vivax/P. ovale/P. malariae. Unlike the widely used circumsporozoite protein-ELISA method the TaqMan assay targets species specific sequence variation in the small subunit ribosomal RNA gene and is therefore, not stage specific. This assay will therefore detect stages of Plasmodium in the blood meal/gut of the mosquito, and to ensure that only sporozoites within the salivary glands are detected, DNA should be extracted from head thorax only and the abdomen removed prior to extraction. The sensitivity and the specificity of the new assay was compared to three alternative PCR approaches and to microscopic dissection of salivary glands in a blind trial of single insect samples that included artificially infected Anopheles stephensi mosquitoes and more than 450 field-collected specimens that had been stored either on silica gel or in ethanol or isopropanol. Tests of analytical sensitivity and the results of the blind trial showed the TaqMan assay to be the most sensitive of the four methods followed by the "gold standard" nested PCR approach and the results generated using these two methods were in good concordance [6]. The TaqMan method was not inhibited by any of the mosquito storage methods.

\section{Resistance to Insecticides}

Current vector control strategies rely heavily on the use of insecticides, most commonly employed as insecticidetreated nets (ITNs) and indoor residual spraying (IRS). Unfortunately, their intensive use has led to the development of resistance and this has the potential to seriously compromise malaria control efforts $[26,27]$. Sensitive detection of resistance is a prerequisite for resistance management strategies aimed at prolonging insecticide life while maintaining sufficient insect control. Bioassay assessment of mosquito vector populations for resistance is an essential component 
TABle 1: Mosquito and Plasmodium specimens suitable for use as controls in VPMT assays that are available from the Malaria Research and Reference Reagent Resource Centre (MR4) http://www.mr4.org/.

\begin{tabular}{|c|c|c|c|}
\hline Applicable TaqMan assay & MR4 reference No. & Species/genotype & Further details \\
\hline Species ID & MRA-495 & An. arabiensis & Genomic DNA from KGB (MRA-339) \\
\hline Species ID & MRA-142 & An. gambiae & Genomic DNA from An. gambiae, G3 strain \\
\hline Species ID & MRA-761 & An. quadriannulatus & from South Africa \\
\hline Plasmodium infection & MRA-102G & Plasmodium falciparum & Genomic DNA from $P$. falciparum 3D7 \\
\hline Plasmodium infection & MRA-341G & Plasmodium vivax & Genomic DNA from P. vivax ONG \\
\hline$K d r$ assay & MRA-762 & An. gambiae/homozygous wild type & $\begin{array}{l}\text { KISUMU strain, considered the insecticide } \\
\text { susceptible standard }\end{array}$ \\
\hline$K d r$ assay & MRA334 & An. gambiae/homozygous for L1014S & $\begin{array}{l}\text { RSP strain, expresses the L1014S kdr } \\
\text { mechanism }\end{array}$ \\
\hline$R d l$ assay & MRA-762 & An. gambiae/homozygous wild type & $\begin{array}{l}\text { KISUMU strain, considered the insecticide } \\
\text { susceptible standard }\end{array}$ \\
\hline$R d l$ assay & MRA-495 & An. arabiensis/homozygous wild type & Genomic DNA from KGB (MRA-339) \\
\hline$R d l$ assay & MRA-116 & An. gambiae/homozygous for A296G & $\begin{array}{l}\mathrm{P}+\mathrm{DLRC}+\mathrm{R}+\text { strain, dieldrin resistant due to } \\
\text { Rdl mutation (Ala296Gly) }\end{array}$ \\
\hline$R d l$ assay & MRA764 & An. arabiensis/homozygous for A296S & $\begin{array}{l}\text { SENN strain, dieldrin resistant due to Rdl } \\
\text { mutation (Ala296Ser) }\end{array}$ \\
\hline
\end{tabular}

of monitoring programmes as it can detect moderate-to-high resistance phenotypes regardless of the underlying resistance mechanism(s). However, the methodology involved can be time consuming, requires live insects in good condition, and often cannot detect resistance at a low frequency. In contrast, mechanism-specific high-throughput diagnostic assays are both more rapid and can detect resistance earlier.

6.1. Detection of Knock down Resistance (kdr). Knockdown resistance $(\mathrm{kdr})$ is a well-characterized mechanism of resistance to DDT and pyrethroid insecticides in many insect species and is caused by point mutation of the target site, the para-type sodium channel. In pyrethroid resistant An. gambiae, two amino acid substitutions at the same position (L1014F and L1014S) have been reported, initially in An. gambiae s.s. [28, 29] and more recently in Anopheles arabiensis [30, 31]. Recently, individuals heterozygous for both the F1014 and S1014 alleles have been reported [3, 32].

Two assays have been developed for the detection of the L1014F or L1014S mutations [4]. Both kdr assays use two probes, the first specific for the wild-type allele is labelled with VIC and the second specific for the mutant allele (F1014 and S1014), is labelled with FAM. In both assays a substantial increase in VIC fluorescence indicates a homozygous wildtype, a substantial increase in FAM fluorescence indicates a homozygous mutant and a usually intermediate increase in both signals indicates a heterozygote (see example in Figure 2). Individuals homozygous for the L1014S mutation display no increase in VIC or FAM fluorescence in the L1014F assay, and vice versa. The sensitivity and specificity of the TaqMan assays was compared to four previously reported techniques for $\mathrm{kdr}$ detection, Allele Specific Polymerase Chain Reaction (AS-PCR), Heated Oligonucleotide Ligation Assay (HOLA), Sequence Specific Oligonucleotide ProbeEnzyme-Linked Immunosorbent Assay (SSOP-ELISA) and PCR-Dot Blot in a blind genotyping trial of 96 single insect samples that included a variety of kdr genotypes and African Anopheline species. The results demonstrated that the realtime TaqMan assays were both the most sensitive (with the lowest number of failed reactions) and the most specific (with the lowest number of incorrect scores) [4].

6.2. Detection of Insensitive Acetylcholinesterase (iAChE). Resistance to organophosphate and carbamate insecticides in Anopheles gambiae can result from insensitivity of the target site enzyme, acetylcholinesterase [33]. Resistance results from a single nucleotide substitution in the ace-1 gene encoding the enzyme, conferring a Glycine to Serine (G119S) amino acid substitution (termed ace-1 $1^{R}$ ) in the oxyanion hole of the enzyme. A TaqMan assay [7] was developed for sensitive detection of this mutation that like the $\mathrm{kdr}$ assays uses two probes, the first labelled with VIC is specific for the wild-type (G119) allele, and the second labelled with FAM is specific for the mutant allele (S119). The TaqMan assay was compared to the existing standard PCR-RFLP method to genotype over 280 samples and proved to be both more sensitive and specific [7].

6.3. Detection of Resistance to Dieldrin ( $R d l$ l). In many insect species, resistance to cyclodiene insecticides is caused by amino acid substitutions at a single residue within the M2 transmembrane region of the $\gamma$-aminobutyric acid (GABA) receptor subunit termed Rdl (resistance to dieldrin) [34]. These mutations have also been shown to confer low levels of cross-resistance to fipronil, a phenylpyrazole insecticide with a similar mode of action to cyclodienes. Two alternative mutations have been reported in An. gambiae sensu lato (s.l.), A296G in An. gambiae s.s. and A296S in An. arabiensis [35]. Two TaqMan assays, one for each of the two alternative mutations, are reported here for the first time. The A296G assay uses two primers, GlyRdlF (5' -TCATATCGTGGGTATCATTTTGGCTAAAT- ${ }^{\prime}$ ) and 


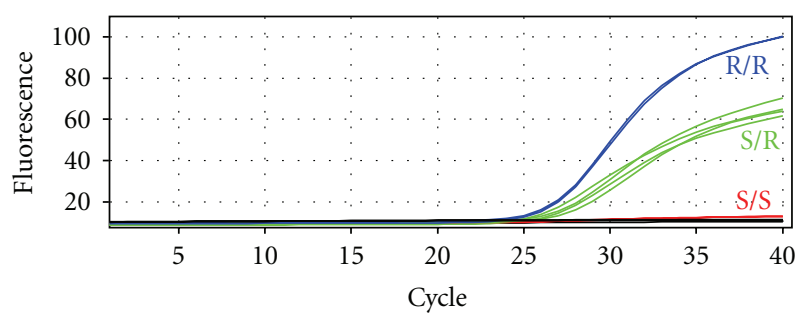

(a) Cycling of FAM probe (phenylalanine)

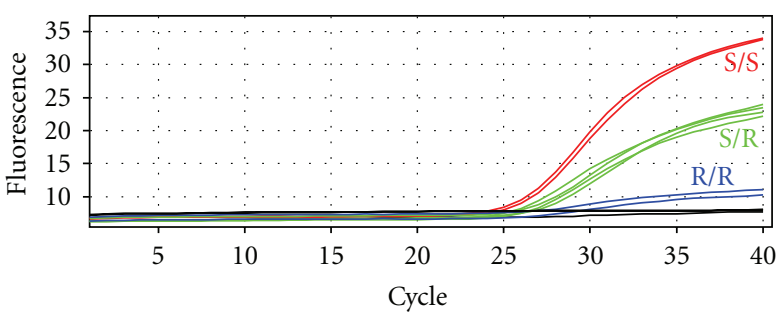

(b) Cycling of VIC probe (leucine)

Figure 2: Real-time TaqMan detection of the L1014F kdr mutation. S: wild-type allele (L1014), R: resistant allele (L1014F).

TABle 2: Approximate cost of each of the VPMT assays per mosquito specimen analysed. In each case costings were calculated in US\$ by using retail prices from US websites. These prices do not include promotional or negotiated discounts or the cost of delivery. All primer/probe/PCR MasterMix costs are from ordering at the largest available scale. Cost calculations include all the plastics required to set up the reactions. For the cost of the kdr and Rdl assays, this is the combined cost of running two assays (i.e., A296G + A296S).

\begin{tabular}{lc}
\hline Assay & Cost in US\$ \\
\hline An. gambiae species ID (2 plex assay) & 0.75 \\
An. gambiae species ID (3 plex assay) & 1 \\
An. funestus species ID & 0.95 \\
Plasmodium detection assay & 0.75 \\
Kdr assay & 1.5 \\
iAChE assay & 0.75 \\
$R d l$ assay & 1.5 \\
\hline
\end{tabular}

GlyRdlR ( $5^{\prime}$-CGACATCAGTGTTGTCATTGTCAAG-3') and two probes, WT1 ( $5^{\prime}$-ACGTGTTGCATTAGG-3') specific for the wild-type allele labelled with VIC and Gly $\left(5^{\prime}\right.$ -ACGTGTTGGATTAGG-3'), specific for the mutant allele (G296) labelled with FAM. The A296S assay uses primers SerRdlF ( $5^{\prime}$-TCATATCGTGGGTATCATTTTGGCTAAAT$\left.3^{\prime}\right)$ and SerRdlR ( $5^{\prime}$-TCGTTGACGACATCAGTGTTGT-3') and two probes, WT2 (5' $5^{\prime}$ TTACACCTAATGCAACACG$\left.3^{\prime}\right)$ specific for the wild-type allele labelled with VIC and Ser ( $5^{\prime}$-CACCTAATGAAACACG-3'), specific for the mutant allele (S296) labelled with FAM. All probes also have a $3^{\prime}$ nonfluorescent quencher and a minor groove binder at the $3^{\prime}$ end. The minor groove binder provides more accurate allelic discrimination by increasing the $T_{M}$ between matched and mismatched probes [36]. PCR reaction conditions are as described for the kdr assay [4] except for temperature cycling conditions for the A296G assay which were 10 minutes at $95^{\circ} \mathrm{C}$ followed by 40 cycles of $95^{\circ} \mathrm{C}$ for 10 seconds and $62^{\circ} \mathrm{C}$ for 45 seconds. In either assay a substantial increase in VIC fluorescence indicates a homozygous wildtype, a substantial increase in FAM fluorescence indicates a homozygous mutant, and a usually intermediate increase in both signals indicates a heterozygote (Figure 3 ). The sensitivity and specificity of the TaqMan assays was examined using serial dilutions of mosquito DNA extracted from individuals of the An. gambiae $\mathrm{P}+\mathrm{DLRC}+\mathrm{R}+$ (homozygous for A296G) and Kisumu strains (wild type), An. arabiensis SENN (homozygous for A296S), and KGB strains (wild type) and An gambiae s.l. samples from Ghana and Cameroon that were heterozygous for A296G or A296S by sequencing. For this DNA preparations were diluted to $20 \mathrm{ng} / \mathrm{ul}$ then serially diluted down to a 1 in $1 \times 10^{4}$ dilution. In this small scale trial of 96 samples, the assay correctly identified all possible genotypes, and the limit of detection for both the A296G and A296S TaqMan assays was a 1 in 10,000 dilution representing 2pg DNA.

\section{Feedback from Field Testing}

Field testing of the VPMT by ourselves and others has taken place in Malawi and South Africa, and VPMT protocols are now being used routinely to support interventions in Equatorial Guinea and southern Africa and for other studies [37-40]. Feedback from end users has enabled us to develop additional guidelines and advice. General advice applicable to the use of all the VPMT protocols is as follow. (1) Include controls in assay runs. We recommend that these include one or more no-template controls and in the case of the An. gambiae s.l. species ID and Plasmodium infection assays a positive control for each probe (e.g., An. gambiae s.s, An. arabiensis and An. quadriannulatus for the 3-plex species ID assay and $P$. falciparum and $P$. vivax for the Plasmodium assay). In the case of the $\mathrm{kdr}, \mathrm{iAChE}$ and Rdl assays a control template for each genotype should be included (e.g., when running the L1014F assay include a L1014F homozygous sample, a wild-type homozygous sample, and a L1014F heterozygous sample). We have found that including these controls greatly facilitates the interpretation of results and generally speeds up the scoring of unknown samples. In this regard Table 1 details relevant control genotypes currently available from the Malaria Research and Reference Reagent Resource Center (MR4), http://www.mr4.org/. (2) We recommend making aliquots of all reagents to avoid repeated freeze-thawing. This is especially important for fluorescently labelled probes which should also be stored out of the light to prevent UV degradation. If probes or reagents need to be transported a thermos flask full of ice and a small amount of salt will keep them cold for up to 24 hours. (3) We have found that the "auto-scale" function of the software that accompanies most real-time PCR machines should be used with caution as this can artificially elevate the fluorescent trace resulting in misscoring. (4) If further 


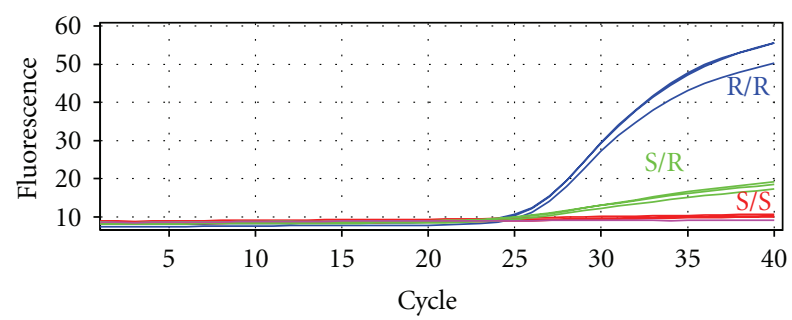

(a) Cycling FAM probe (serine)

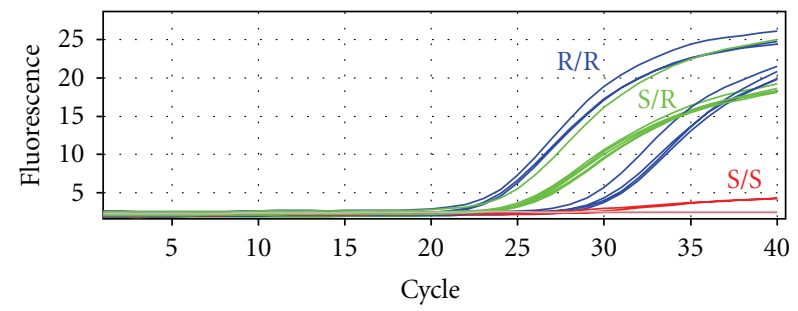

(c) Cycling FAM probe (glycine)

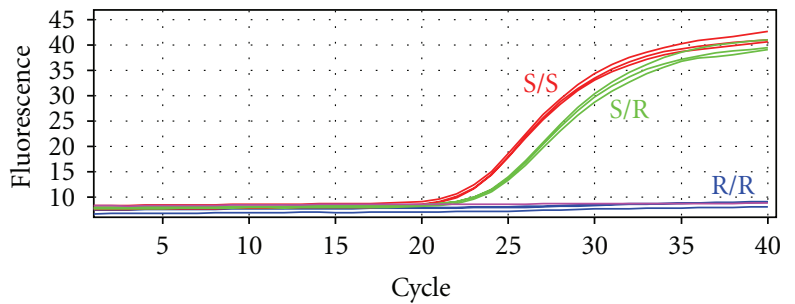

(b) Cycling VIC probe (alanine)

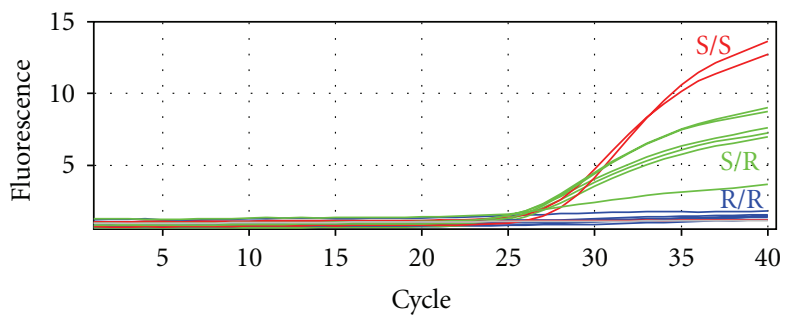

(d) Cycling VIC probe (alanine)

Figure 3: Real-time TaqMan detection of the Rdl mutation in Anopheles gambiae and Anopheles arabiensis. (a) and (b) Detection of the A296S mutation. (c) and (d) Detection of the A296G mutation.

assay optimisation is required the annealing temperature, the concentration of probes in the reaction and number of temperature cycles are good parameters to alter in attempts to enhance sensitivity or reduce nonspecific signals. (5) We have found the TaqMan protocols to be largely unaffected by variation in DNA extraction methodology and a range of popular extraction methods can be used to good effect [4] the exception to this rule is the Plasmodium assay (discussed below).

7.1. Specific Advice on the An. gambiae S.l. Species Complex Identification Assay. The 3-plex species identification assay was initially run on a Corbett rotorgene PCR machine with an annealing/extension time of $60^{\circ} \mathrm{C}$. At this temperature the probes Aa and Aq (Cy5- and 6FAM-labelled) showed specific amplification of An. arabiensis and An. quadriannulatus/An. melas/An. merus respectively. However while the probe Ag (VIC-labelled) also gave sensitive detection of An. gambiae s.s., when An. quadriannulatus, An. melas or An. merus DNAs were tested a low-level "background" fluorescence signal was observed, presumably from nonspecific binding of this probe. This could be eliminated by increasing the annealing/extension temperature to $66^{\circ} \mathrm{C}$ and lowering the final probe concentration in the PCR from 200 to $80 \mathrm{nM}$. However, when this assay was run on an alternative PCR machine (Chromo4, Bio-Rad) using the modified conditions and white PCR tubes, the same low-level "background" was seen once more. Increasing the annealing/extension temperature to $67^{\circ} \mathrm{C}$ and lowering the final probe concentration in the PCR from 200 to $50 \mathrm{nM}$ showed a degree of improvement but did not completely eliminate the background signal. In practice, this background does not inhibit scoring as an $A n$. quadriannulatus, An. melas, or An. merus specimen shows a strong signal in the Cy5 channel, while a true Anopheles gambiae s.s. individual only shows signal in the VIC channel.
7.2. Specific Advice on the Plasmodium Detection Assay. The Plasmodium detection assay was originally optimised using genomic DNA templates, and carrying out 45 cycles of PCR. When tests were carried out on blood-fed mosquitoes extracted by crude methods (such as boiling whole mosquitoes in sucrose buffer), a small amount of nonspecific fluorescence was sometimes seen after 40 cycles. For this reason, we recommend restricting the number of cycles in PCR to 40. In addition, we have had reports that the Plasmodium assay is particularly sensitive to contamination of reagents and so it is especially important to aliquot reagents, to use filter sterilised water to make up reactions and filter tips to dispense reaction components and DNA.

7.3. Specific Advice on the Detection of Knock down Resistance $(k d r)$ Assay. If separate runs have been performed on the same samples for L1014F and L1014S, when interpreting the results it is useful to have two copies of the real-time PCR machine software open on the computer at once. In one copy open the L1014F run and in the other open the L1014S run. The same sample can then be viewed in each assay by moving between the two runs/copies of the software. This helps to rapidly assign each sample a genotype. Alternatively, if the east and west assays were run together on the same samples, the same approach can be followed using a single copy of the software.

In our experience, distinguishing between L1014F homozygous and heterozygous genotypes can seem less obvious than scoring other genotypes because a L1014F homozygous genotype shows a very low-level signal in the VIC channel rather than a completely flat line (as with a no-template control) (see Figure 2). In addition, the signal strength from the FAM and VIC probes can be different. Running controls of the two genotypes is very helpful and allows for a direct comparison with unknown samples. 
In our experiments, to date, when we have run the assay on DNA extracted from a single leg, we have obtained mixed results (possibly due to the low quality/concentration of DNA extracted), and, therefore, we cannot recommend the TaqMan kdr assays for this approach.

\section{Summary and Future Development}

Because the VPMT protocols developed to date are based on a closed-tube approach, requiring a single step to achieve results, they are simple, rapid to run, and high-throughput. The results of the genotyping trials carried out in the development of the various assays has also shown that the platform on which they are based is robust, sensitive and specific. The cost of each assay per mosquito analysed is shown in Table 2 and is generally comparable, or in some cases lower, than alternative methods. One disadvantage of the TaqMan assay is the initial cost of the real-time PCR machine required, which is in the region of US\$19,000 to 25,000, compared to the startup cost of conventional PCR of around US\$10,000 [4]. Although the price of real-time PCR machines is falling, and in the future is likely to become comparable to standard thermocyclers, this may currently represent an obstacle to full uptake of these protocols in resource-poor malaria-endemic countries. Perhaps the most significant limitation of the current VPMT is that, although each individual assay is comparable or lower in cost than alternatives, to screen an individual mosquito for every single trait described in this paper is still expensive. We are investigating the possibility of incorporating the current VPMT assays into a single highly multiplexed platform that would allow users to screen for all traits in a single test. A possible platform that is a suitable candidate for such a role is optical thin-film biosensors. Thin-film biosensor chips are capable of transducing specific molecular interactions into signals that can be visualized by the naked eye or by simple digital-imaging systems because mass deposited on the thinfilm surface by enzymatic catalysis alters the wavelength of light reflected by the optical layer resulting in a perceived colour change on the surface [41]. The assay has been described for SNP detection for several animal and plant species and is reported to be robust, exhibit high sensitivity and specificity, and is flexible from low to high throughput [42-44]. We are currently examining the feasibility of using this platform for a multiplexed version of the VPMT.

\section{References}

[1] World Health Organization, http://www.who.int/heli/risks/ risks/vectors/vector/en/index.html.

[2] J. Pinto, A. Lynd, N. Elissa et al., "Co-occurrence of East and West African $k d r$ mutations suggests high levels of resistance to pyrethroid insecticides in Anopheles gambiae from Libreville, Gabon," Medical and Veterinary Entomology, vol. 20, no. 1, pp. 27-32, 2006.

[3] K. Verhaeghen, W. Van Bortel, P. Roelants, T. Backeljau, and M. Coosemans, "Detection of the East and West African $k d r$ mutation in Anopheles gambiae and Anopheles arabiensis from Uganda using a new assay based on FRET/Melt Curve analysis," Malaria Journal, vol. 5, article no. 16, 2006.

[4] C. Bass, D. Nikou, M. J. Donnelly et al., "Detection of knockdown resistance $(k d r)$ mutations in Anopheles gambiae: a comparison of two new high-throughput assays with existing methods," Malaria Journal, vol. 6, article no. 111, 2007.

[5] J. Hemingway, B. J. Beaty, M. Rowland, T. W. Scott, and B. L. Sharp, "The Innovative Vector Control Consortium: improved control of mosquito-borne diseases," Trends in Parasitology, vol. 22, no. 7, pp. 308-312, 2006.

[6] C. Bass, D. Nikou, A. M. Blagborough et al., "PCR-based detection of Plasmodium in Anopheles mosquitoes: a comparison of a new high-throughput assay with existing methods," Malaria Journal, vol. 7, article no. 177, 2008.

[7] C. Bass, D. Nikou, J. Vontas, M. S. Williamson, and L. M. Field, "Development of high-throughput real-time PCR assays for the identification of insensitive acetylcholinesterase $\left(\right.$ ace $\left.-1^{R}\right)$ in Anopheles gambiae," Pesticide Biochemistry and Physiology, vol. 96, no. 2, pp. 80-85, 2010.

[8] C. Bass, M. S. Williamson, and L. M. Field, "Development of a multiplex real-time PCR assay for identification of members of the Anopheles gambiae species complex," Acta Tropica, vol. 107 , no. 1, pp. 50-53, 2008.

[9] C. Bass, M. S. Williamson, C. S. Wilding, M. J. Donnelly, and L. M. Field, "Identification of the main malaria vectors in the Anopheles gambiae species complex using a TaqMan real-time PCR assay," Malaria Journal, vol. 6, article no. 155, 2007.

[10] S. B. Vezenegho, C. Bass, M. Puinean et al., "Development of multiplex real-time PCR assays for identification of members of the Anopheles funestus species group," Malaria Journal, vol. 8 , no. 1 , article no. $282,2009$.

[11] K. J. Livak, "Allelic discrimination using fluorogenic probes and the 5' nuclease assay," Genetic Analysis-Biomolecular Engineering, vol. 14, no. 5-6, pp. 143-149, 1999.

[12] M. Moreno, J. Cano, S. Nzambo et al., "Malaria Panel Assay versus PCR: detection of naturally infected Anopheles melas in a coastal village of Equatorial Guinea," Malaria Journal, vol. 3, article no. 20, 2004.

[13] J. M. Léong Pock Tsy, J. B. Duchemin, L. Marrama et al., "Distribution of the species of the Anopheles gambiae complex and first evidence of Anopheles merus as a malaria vector in Madagascar," Malaria Journal, vol. 2, article no. 1, 2003.

[14] M. Coetzee, "Distribution of the African malaria vectors of the Anopheles gambiae complex," American Journal of Tropical Medicine and Hygiene, vol. 70, no. 2, pp. 103-104, 2004.

[15] M. Coetzee, M. Craig, and D. Le Sueur, "Distribution of African malaria mosquitoes belonging to the Anopheles gambiae complex," Parasitology Today, vol. 16, no. 2, pp. 7477, 2000.

[16] G. B. White, “Anopheles bwambae sp. n., a malaria vector in Semliki Valley, Uganda, and its relationships with other sibling species of the An. gambiae complex," Systematic Entomology, vol. 10, pp. 501-522, 1985.

[17] E. D. Walker, A. R. Thibault, A. P. Thelen et al., "Identification of field caught Anopheles gambiae s.s. and Anopheles arabiensis by TaqMan single nucleotide polymorphism genotyping," Malaria Journal, vol. 6, article no. 23, 2007.

[18] M. K.N. Lawniczak, S. J. Emrich, A. K. Holloway et al., "Widespread divergence between incipient Anopheles gambiae species revealed by whole genome sequences," Science, vol. 330, no. 6003, pp. 512-514, 2010.

[19] D. E. Neafsey, M. K. N. Lawniczak, D. J. Park et al., "SNP genotyping defines complex gene-flow boundaries among 
african malaria vector mosquitoes," Science, vol. 330, no. 6003, pp. 514-517, 2010.

[20] M. T. Gillies and M. Coetzee, "Supplement to the Anophelinae of Africa South of the Sahara," Publications of the South African Institute for Medical Research, no. 55, 1987.

[21] M. T. Gillies and B. De Meillon, "The Anophelinae of Africa south of the Sahara," Publications of the South African Institute for Medical Research, no. 54, 1968.

[22] R. E. Harbach, "The classification of genus Anopheles (Diptera: Culicidae): a working hypothesis of phylogenetic relationships," Bulletin of Entomological Research, vol. 94, no. 6, pp. 537-553, 2004.

[23] M. Coetzee and D. Fontenille, "Advances in the study of Anopheles funestus, a major vector of malaria in Africa," Insect Biochemistry and Molecular Biology, vol. 34, no. 7, pp. 599605, 2004.

[24] B. De Meillon, G. Van Eeden, L. Coetzee et al., "Observations on a species of Anopheles funestus subgroup, a suspected exophilic vector of malaria parasites in north-eastern Transvaal, South Africa," Mosquito News, vol. 37, pp. 657-661, 1977.

[25] T. J. Wilkes, Y. G. Matola, and J. D. Charlwood, “Anopheles rivulorum, a vector of human malaria in Africa," Medical and Veterinary Entomology, vol. 10, no. 1, pp. 108-110, 1996.

[26] K. Hargreaves, L. L. Koekemoer, B. D. Brooke, R. H. Hunt, J. Mthembu, and M. Coetzee, "Anopheles funestus resistant to pyrethroid insecticides in South Africa," Medical and Veterinary Entomology, vol. 14, no. 2, pp. 181-189, 2000.

[27] R. N'Guessan, V. Corbel, M. Akogbéto, and M. Rowland, "Reduced efficacy of insecticide-treated nets and indoor residual spraying for malaria control in pyrethroid resistance area, Benin," Emerging Infectious Diseases, vol. 13, no. 2, pp. 199-206, 2007.

[28] H. Ranson, B. Jensen, J. M. Vulule, X. Wang, J. Hemingway, and F. H. Collins, "Identification of a point mutation in the voltage-gated sodium channel gene of Kenyan Anopheles gambiae associated with resistance to DDT and pyrethroids," Insect Molecular Biology, vol. 9, no. 5, pp. 491-497, 2000.

[29] D. Martinez-Torres, F. Chandre, M. S. Williamson et al., "Molecular characterization of pyrethroid knockdown resistance ( $k d r)$ in the major malaria vector Anopheles gambiae s.s," Insect Molecular Biology, vol. 7, no. 2, pp. 179-184, 1998.

[30] A. Diabaté, T. Baldet, F. Chandre et al., "First report of a $k d r$ mutation in Anopheles arabiensis from Burkina Faso, West Africa," Journal of the American Mosquito Control Association, vol. 20, no. 2, pp. 195-196, 2004.

[31] A. D. Stump, F. K. Atieli, J. M. Vulule, and N. J. Besansky, "Dynamics of the pyrethroid knockdown resistance allele in western Kenyan populations of Anopheles gambiae in response to insecticide-treated bed net trials," American Journal of Tropical Medicine and Hygiene, vol. 70, no. 6, pp. 591-596, 2004.

[32] J. Etang, E. Fondjo, F. Chandre et al., "Short report: first report of knockdown mutations in the malaria vector Anopheles gambiae from Cameroon," American Journal of Tropical Medicine and Hygiene, vol. 74, no. 5, pp. 795-797, 2006.

[33] M. Weill, G. Lutfalla, K. Mogensen et al., "Erratum: insecticide resistance in mosquito vectors (Nature (2003) 423 (136137))," Nature, vol. 425, no. 6956, p. 366, 2003.

[34] R. H. Ffrench-Constant, N. Anthony, K. Aronstein, T. Rocheleau, and G. Stilwell, "Cyclodiene insecticide resistance: from molecular to population genetics," Annual Review of Entomology, vol. 45, pp. 449-466, 2000.
[35] W. Du, T. S. Awolola, P. Howell et al., "Independent mutations in the Rdl locus confer dieldrin resistance to Anopheles gambiae and An. arabiensis," Insect Molecular Biology, vol. 14, no. 2, pp. 179-183, 2005.

[36] I. Afonina, M. Zivarts, I. Kutyavin, E. Lukhtanov, H. Gamper, and R. B. Meyer, "Efficient priming of PCR with short oligonucleotides conjugated to a minor groove binder," Nucleic Acids Research, vol. 25, no. 13, pp. 2657-2660, 1997.

[37] T. Mzilahowa, A. J. Ball, C. Bass et al., "Reduced susceptibility to DDT in field populations of Anopheles quadriannulatus and Anopheles arabiensis in Malawi: evidence for larval selection," Medical and Veterinary Entomology, vol. 22, no. 3, pp. 258-263, 2008.

[38] U. Ramphul, T. Boase, C. Bass, L. M. Okedi, M. J. Donnelly, and P. Müller, "Insecticide resistance and its association with target-site mutations in natural populations of Anopheles gambiae from eastern Uganda," Transactions of the Royal Society of Tropical Medicine and Hygiene, vol. 103, no. 11, pp. 1121-1126, 2009.

[39] F. C. Ridl, C. Bass, M. Torrez et al., "A pre-intervention study of malaria vector abundance in Rio Muni, Equatorial Guinea: their role in malaria transmission and the incidence of insecticide resistance alleles," Malaria Journal, vol. 7, article no. 194, 2008.

[40] A. Lynd, D. Weetman, S. Barbosa et al., "Field, genetic, and modeling approaches show strong positive selection acting upon an insecticide resistance mutation in Anopheles gambiae s.s," Molecular Biology and Evolution, vol. 27, no. 5, pp. 11171125, 2010.

[41] X. B. Zhong, R. Reynolds, J. R. Kidd et al., "Single-nucleotide polymorphism genotyping on optical thin-film biosensor chips," Proceedings of the National Academy of Sciences of the United States of America, vol. 100, no. 20, pp. 11559-11564, 2003.

[42] S. Bai, J. Zhang, S. Li et al., "Detection of six genetically modified maize lines using optical thin-film biosensor chips," Journal of Agricultural and Food Chemistry, vol. 58, no. 15, pp. 8490-8494, 2010.

[43] S. Bai, J. Zhao, Y. Zhang et al., "Rapid and reliable detection of 11 food-borne pathogens using thin-film biosensor chips," Applied Microbiology and Biotechnology, vol. 86, no. 3, pp. 983990, 2010.

[44] K. Nakamoto, J. R. Kidd, R. D. Jenison et al., "Genotyping and haplotyping of CYP2C19 functional alleles on thin-film biosensor chips," Pharmacogenetics and Genomics, vol. 17, no. 2, pp. 103-114, 2007. 


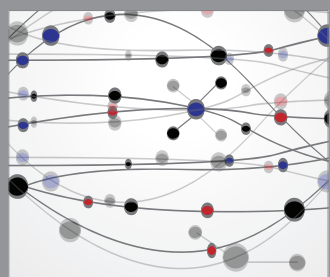

The Scientific World Journal
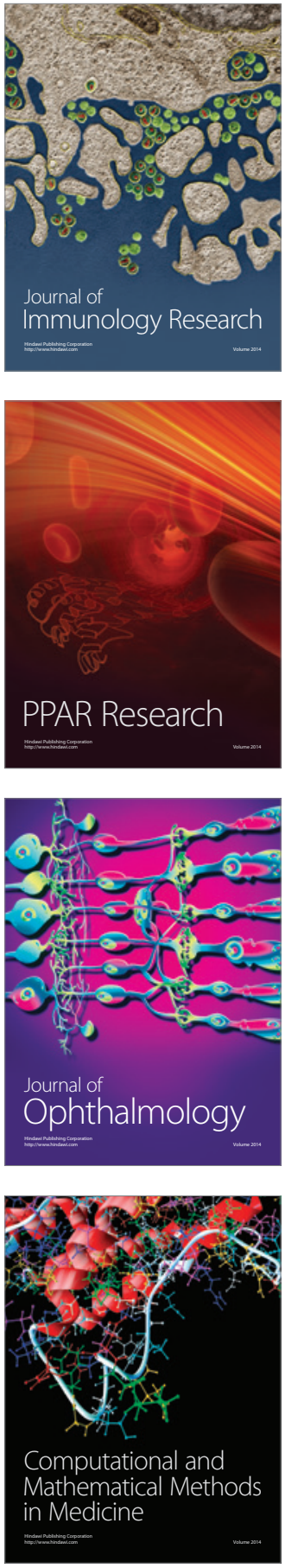

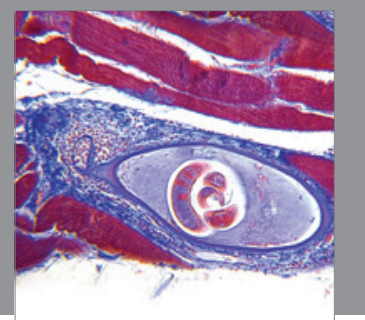

Gastroenterology

Research and Practice
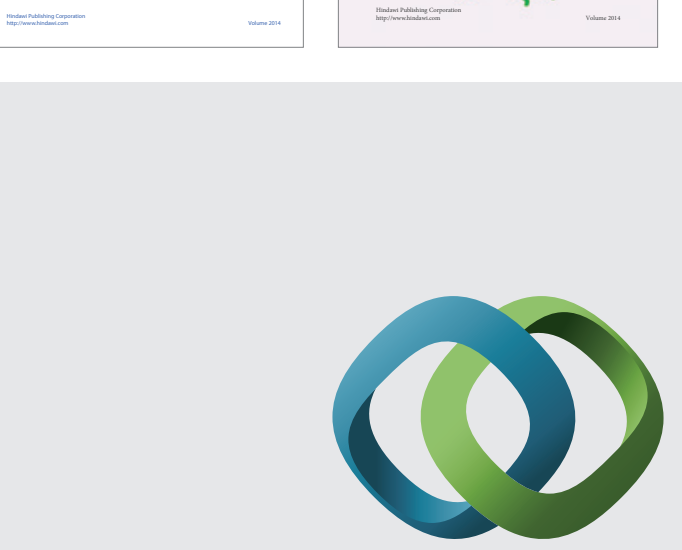

\section{Hindawi}

Submit your manuscripts at

http://www.hindawi.com
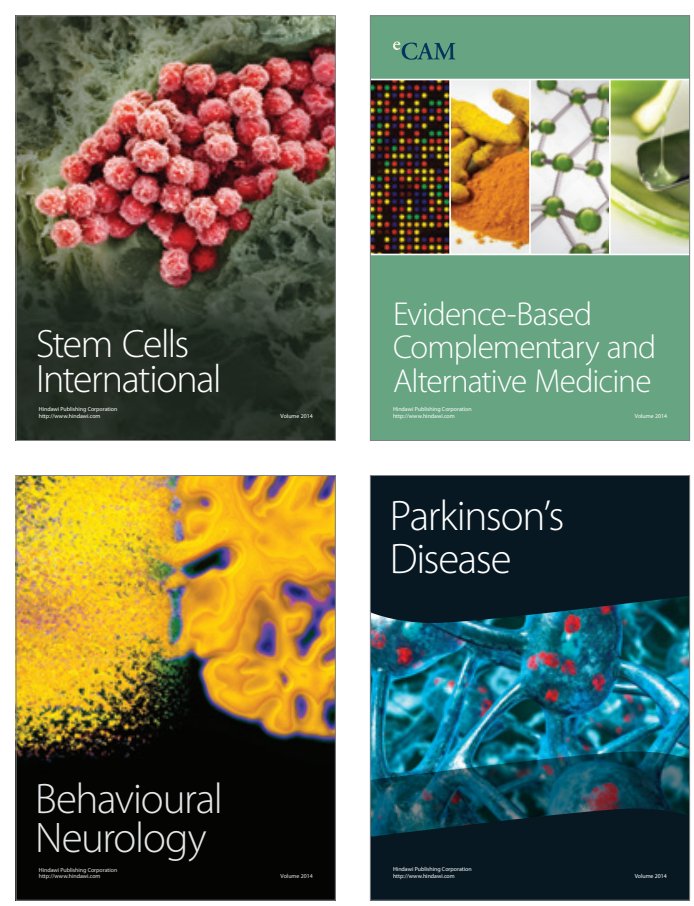

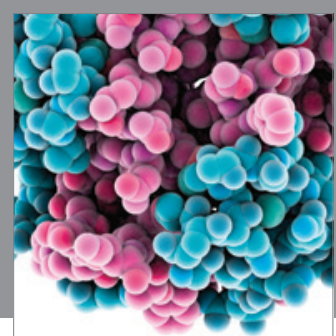

Journal of
Diabetes Research

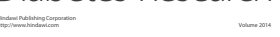

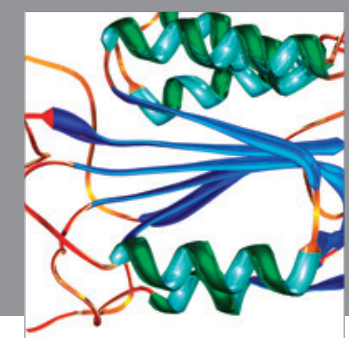

Disease Markers
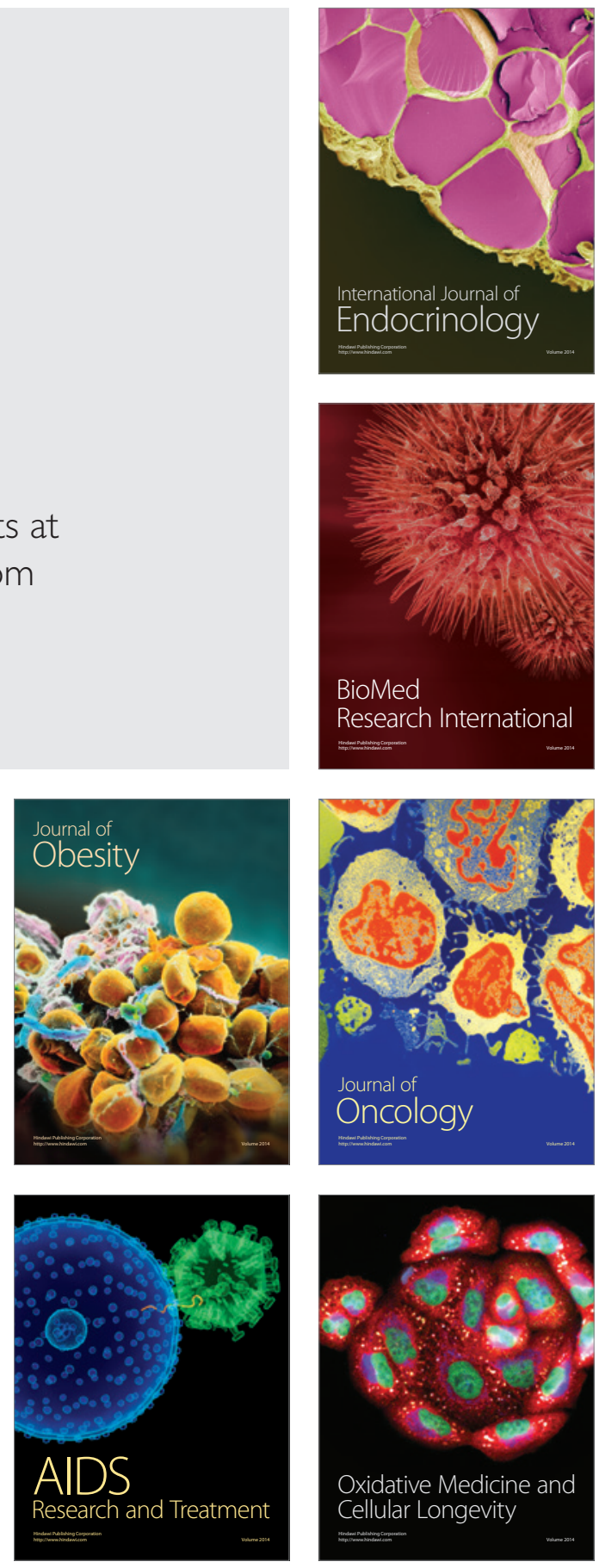